\title{
A Volumetric Virtual Environment for Catheter Insertion Simulation
}

\author{
Antonio Zorcolo, Enrico Gobbetti, Gianluigi Zanetti, and Massimiliano Tuveri \\ CRS4 \\ Center for Advanced Studies, Research, and Development in Sardinia \\ VI Strada Ovest, Z.I. Macchiareddu, C.P. 94, I-09010 Uta (CA), Italy \\ E-mail: $\{$ zarco, gobbetti, zag, mtuveri\}@ers4.it, \\ WWW: http: : //www.crs4.it/vvr
}

\begin{abstract}
We present an experimental catheter insertion simulation system that provides users co-registered haptic and head-tracked stereoscopic visual feedback. The system works on patient-specific volumetric data acquired using standard medical imaging modalities. The actual needle insertion operation is simulated for individual patients, rather than being an example of a model surgical procedure on standard anatomy. Patient specific features may thus be studied in detail by the trainees, overcoming one of the major limitations of current training techniques.
\end{abstract}

\section{Introduction}

The insertion of a catheter into a vessel (artery or vein) is one of the most common procedures in clinical practice. This procedure has an especially important role during percutaneous cardiac catheterization and every time central venous access is required. Precise catheter insertion requires a perfect knowledge of the three-dimensional development of vessels and a high level of dexterity during vessel puncture, which is only attainable after considerable practice. If badly performed, catheter insertion can result in undue patient harm, and inadequate training in this technique is a leading cause of hospital-acquired infections. Computer simulation is expected to be useful in improving training beyond the limitations of the current methods of in vivo practice and usage of artificial physical models [13].

In this paper, we present an experimental catheter insertion simulation system that provides users co-registered visual and haptic feedback. The system works on patientspecific volumetric data acquired using standard medical imaging modalities. The actual needle insertion operation is simulated for individual patients, rather than being an example of a model surgical procedure on standard anatomy. Patient specific features may thus be studied in detail by the trainees, overcoming one of the major limitations of current training techniques.

The rest of the paper is organized as follows. Section 2 provides an overview of the system, section 3 concentrates on data acquisition, section 4 details the visual rendering techniques used, while section 5 discusses tissue penetration simulation and haptic rendering. The paper concludes with a discussion of the results obtained and a view of future work. 


\section{System Overview}

In a lot of common procedures, catheters are inserted percutaneously into deep vessels (e.g. when inserting central venous catheters (CVCs) into deep veins such as the subclavian, jugular, or femoral veins). In those cases, a needle with a syringe attached is placed into the vessel. When the vessel is reached, a guidewire is passed through the needle, the needle is removed, and a dilator and sheath are threaded over the wire. Finally, the dilator is removed and the catheter positioned. The first part of the operation, needle insertion, is difficult to learn, and requires a combination of visual and tactile skills, to identify the needle insertion point and control needle position and orientation during penetration. It is thus important for a training system to provide co-registered visual and haptic responses.

Our system enhances touch perception with visual perception by using head-tracked stereoscopic viewing and a custom made display system which provides hand-immersed interaction (see figure 1). The user looks into a mirror through shutter glasses and perceives the virtual image within the workspace of a PHANToM haptic device which controls needle insertion.
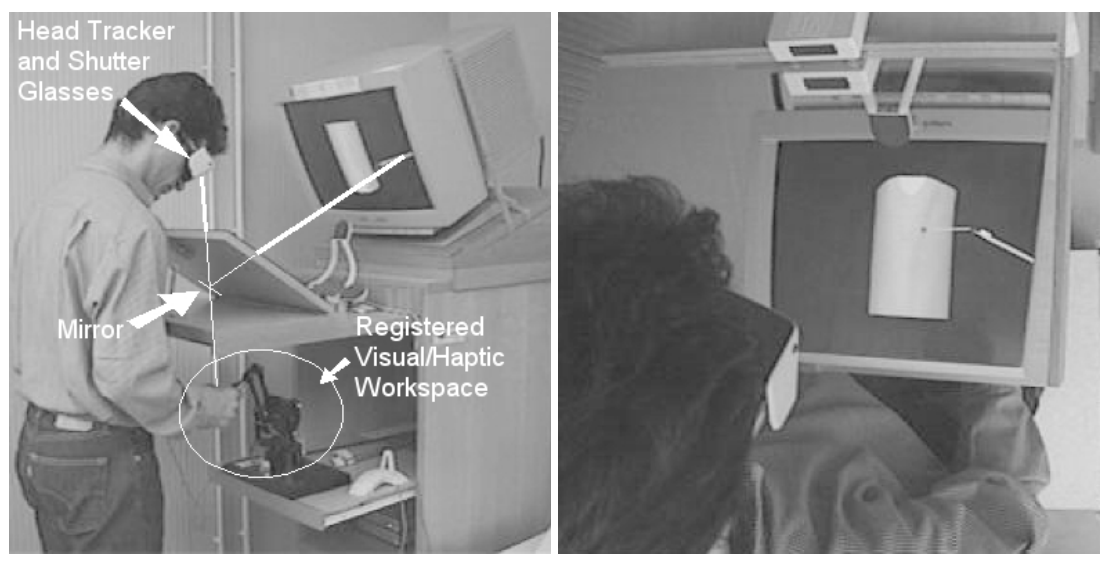

Fig. 1. Haptic Workbench Configuration. The user looks into a mirror through shutter glasses and perceives the virtual image within the workspace of a PHANToM haptic device which controls needle insertion. The user can thus manipulate and feel the needle with his hands without obscuring the display.

By co-registering physical and virtual spaces beforehand and using stereoscopic projections that dynamically follow head movements, the virtual patient is made to appear at a fixed physical position on a virtual surgical table. This produces, without resorting to an head-mounted display, a combined haptic and stereoscopic view of a virtual volume in which users can manipulate the needle with their hands without obscuring the display (see figure 1). During the insertion procedure the system provides a combined visual and haptic perception of penetration and positional deviation of the inserted catheter. 
The system runs on a single two processor Silicon Graphics Onyx IR. As visual feedback and force feedback have different frequency requirements dictated by the human perceptual system, we have implemented our system using multiprocessing and shared memory communication. One processor is reserved to force feedback computation, while the second processor is used for visual rendering and other application tasks. Force feedback computation works at $1 \mathrm{KHz}$, while stereo visual feedback is provided at 10-20 frames per second, depending on the complexity of the dataset. The use of shared memory for inter-process communication reduces the latency of the application, increasing the spatio-temporal realism of the simulation.

Given the characteristics of the needle insertion procedure, we have decided to build the simulator around a volumetric model of the patient anatomy, providing both visual and haptic feedback using a direct volume rendering approach, without resorting to surface reconstruction. This makes it possible, in particular, to interactively analyze a patient's anatomy and to visually control the position of the needle during insertion using semi-transparent mappings.

\section{Dataset Acquisition and Classification}

In clinical practice it is becoming commonplace to take a consecutive series of patient scans using high resolution MRI and spiral CT. Patients will undergo a specific surgical intervention only after careful image processing and pre-operative planning using this data has taken place. Our system exploits the large amounts of available quantitative information on a patient's internal structures to build patient-specific volumetric models. The medical data we use is stored on our CTN image server [15] and it is provided by research collaborations with clinical research institutions. Data transmission between the hospitals and our PACS is usually performed using DICOM.

Our needle insertion simulator takes directly as input datasets in the DICOM format and can work both on raw and segmented data. Our segmentation system, used to differentiate among the tissues in the dataset, combines a 2D edge follower, snakes [8], and balloon models [3]. When working on raw data, Drebin classification [5] is used instead to map voxel values to tissue identifiers. In all cases, the input to the simulator becomes thus a 3D array of tissue identifiers, and a lookup table is used to map this data to the optical and physical tissue properties required for visual and dynamic simulation. The optical properties lookup table contains color and opacity assigned to each tissue, while the dynamic simulation lookup table contains tissue penetration and surface resistance parameters (i.e. static and dynamic friction coefficients and surface break forces).

\section{Visual Rendering}

In direct volume rendering, image computation is based on sampling optical properties associated to volume data and then compositing the samples along each viewing ray. To support hand-immersed interaction, we have to produce at interactive rates perspective stereo pairs combining volumetric rendering of the anatomy with geometric rendering of the needle. To meet performance constraints, we have chosen to directly render the volumes via back-to-front volume slices composition, exploiting texture mapping and 
alpha-blending on high-end graphics accelerators for the sampling and compositing tasks [11,6]. Z-buffer and alpha compositions are used to combine the image of the translucent volume with the image of the opaque needle.

In our implementation, based on OpenGL 3D texturing with Silicon Graphics extensions, the volume is decomposed into bricks, which are then rendered back-to front. Each brick is used to store the values of the input scalar field (raw data or classified tissues identifiers) into a scalar 3D texture and is dimensioned taking into account the hardware constraints (i.e., on our machine, power-of-two dimensions, memory footprint limited to $64 \mathrm{MB}$, dynamic range limited to 16 bits per texel). To render each brick, textured polygons orthogonal to the viewing direction are generated and rendered back-tofront. As our head tracked viewing system requires off-center perspective projections, sampling polygons tessellate spheres centered at the viewing position. To map the values in the texture map into the RGBA quadruples used for the compositing operation, we use a texture color lookup table, a Silicon Graphics extension to OpenGL which makes it possible to map the filtered colors resulting from texturing and pass the result to the texture environment function to be applied to pixel fragments.

Several recent papers describe similar uses of 3D texture mapping hardware for direct volume rendering $[4,2,7,14,9]$. Our major improvements are surface shape enhancement and variable sampling. These aspects are detailed in the following sections.

\subsection{Surface Shape Enhancement}

One of the major limitations of the classic texture-based approaches to volume rendering is the inability to efficiently implement surface illumination models, as texture lookup is based only on data values and not on gradient information. Various authors have proposed alternative techniques to support direct volume rendering with shading via three-dimensional textures (see, e.g. [14]), but this comes at the expense of performance and/or texture memory overhead. As we are dealing with large datasets and have very stringent performance requirements, we have implemented in our renderer a simple color map technique for enhancing tissue surface shapes (see figure 2).

Our technique is based on the fact that when the lighting direction $\mathbf{I}$ is coincident with the viewing direction $\mathbf{v}$ (i.e. the light is positioned on the viewer's head), the angle between $\mathbf{I}$ and the normal $\mathbf{n}$ to the surface separating different tissues depends only on the position of the tissue boundaries on two adjacent slices, once chosen the distance between slices. For example, $\mathbf{n}$ is perpendicular to $\mathbf{l}$ if the boundaries appear super imposed, while it has an angle of 45 degrees with $\mathbf{I}$ when the distance between boundaries is equal to the slice distance.

Because the classification function gives a finite thickness and a decreasing opacity to the boundaries, the surface color is due to the contribution of many boundaries when they are nearly super imposed and is proportional to the sine of the angle between $\mathbf{n}$ and I. Therefore, the application of a color shading to the transition zones of the mapping function leads to a surface shading depending on the angle between $\mathbf{n}$ and $\mathbf{l}$. Moreover, the response to lighting of the surface depends on its strength, which is also defined by the slope of the mapping function in this zone. We thus simulate surface shading by modifying the color lookup table in the transition zone between tissues. The function which is used is the following: 


$$
C_{f}=C_{0}\left[1-\frac{m_{2}-v}{m_{2}-M_{1}}\left(1-\cos \alpha^{\gamma}\right)\right]
$$

where $C_{0}$ and $C_{f}$ are, respectively, the color assigned to the tissue in the original lookup table and the final color, $M_{1}$ and $m_{2}$ delimit the transition zone between the tissues, $v$ is the current voxel value, $\alpha$ determines the slope of the mapping function, and $\gamma$ is a parameter determining the strength of the shading effect (see figure 2).

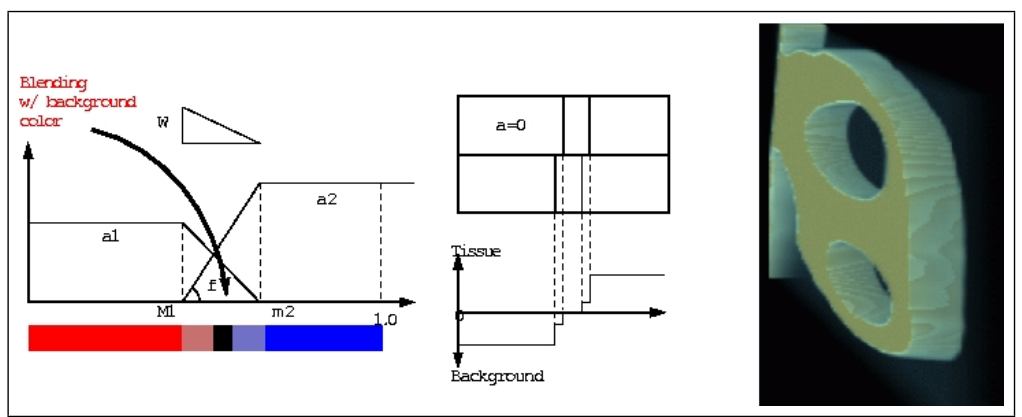

Fig. 2. Surface Enhancement. The left image illustrates the color map techniques. The right image presents a volume rendered view of a section of a carotid artery. The simulated shading enhances surface boundaries.

\subsection{Variable Sampling}

Both image quality and rendering speed are directly related to the number of slices used to sample the volume. The more slices are rendered, the more accurate the results, but the longer the rendering time. Given the timing constraints of our simulation application, it is important to produce good quality images without using an excessively large number of slices, which indicates that the rendering algorithm should concentrate slices where most needed.

Since in perspective rendering the importance of the contribution of the slices to the final image diminishes with their distance to the viewer, a good strategy is to space the slices proportionally to the inverse of that distance. With this approach spatial resolution is increased close to the viewer, making close-up inspection more precise while reducing the details in the background. This is obviously appropriate for needle insertion, where the important details of the anatomy are located near the needle insertion point.

As emission/absorption varies non linearly with thickness [10], special care has to be taken to correctly compute the contribution of each slice. In our renderer, we associate to each slice a thickness which depends on slice spacing. Texture color lookup tables, a Silicon Graphics extension to OpenGL, are then used to define opacity values that depend on slice thickness (see figure 3). Serra et al. also use variable slice spacing, but without opacity correction, thus introducing notable artifacts [12]. 


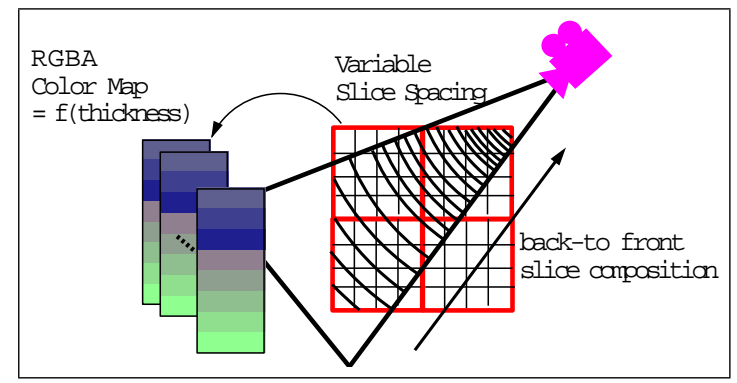

Fig. 3. Variable Sampling. Texture color lookup tables, a Silicon Graphics extension to OpenGL, are used to define opacity values that depend on slice thickness.

\section{Needle insertion simulation and haptic feedback}

Force computation is based on sampling along the needle mechanical properties associated to volume data, reconstructing a local model of the anatomy from the samples, and applying a simplified physical model of tissue deformation and resistance to penetration. Given the limited interaction between tissues and needle during penetration, we have decided to implement a physical deformation model that only updates the local model of the anatomy, leaving the shared volumetric model unchanged. With this approach, we maintain at each step of the simulation an equilibrium state for the needle, which includes position, orientation, and penetrated tissues, and compute reaction forces based on the variation between that state and the state of the externally controlled needle. Force computation depends on tissue parameters (static and dynamic friction coefficients, surface break force) and needle shape parameters (length, radius, angle of attack), which are set using a user-interface panel. The subsystem that updates the needle position in response to hand movements and computes reaction forces is implemented as a state machine, that differentiates between free movement, tissue penetration, and surface piercing.

\subsection{Free movement}

As the PHANToM is a three degrees-of-freedom force-feedback device, no torque can be returned to the user controlling the needle. Thus, we have chosen not to allow the user to firmly grasp the needle but only to hold it at its endpoint, much as a tailor using a thimble. We attach the tip of the PHANToM stylus to the endpoint of the needle and reconstruct its movement from the trajectory of that point. When the needle is free, i.e. when looking for the insertion point before starting the operation, the needle position and orientation are directly controlled by the PHANToM manipulator.

\subsection{Surface contact and piercing}

When the needle tip first collides with the body surface we impose that it stays attached to the collision point, as we consider the collision point to be a micro-hole in the tissue 
surface (see figure 4). Contact continues while the dot product of the exerted force and the surface normal remains negative, i.e. as long as the needle is pushing towards the surface. Tissue surface reaction force is always opposite to the exerted force and applied to the needle tip. Initially we tried to model tissues with a simple spring-damper model. What we observed, manipulating some medical datasets, was a good response applying small forces but an excessive deformation with high ones. So, to take into account tissue stiffening induced by large deformations, we choose to implement the incremental viscoelastic model developed by Berret et al. [1].

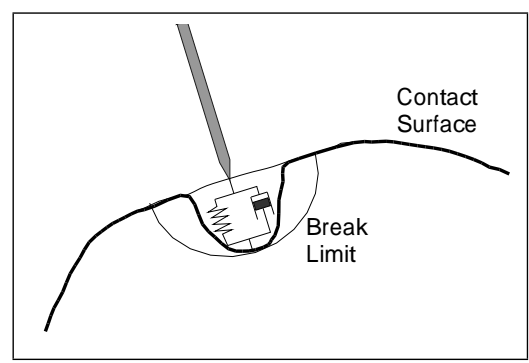

Fig. 4. Surface contact and piercing. Contact continues while the dot product of the exerted force and the surface normal remains negative. The surface is pierces when the exerted force is larger than the break limit.

As we assign at each tissue a surface break force, we can decide when the surface is pierced and the needle enters inside tissue. Once this happens, the system switches state, and stores the needle insertion position and orientation as the equilibrium state. Every subsequent movement of the PHANToM stylus forces the needle to diverge from that orientation (i.e. the needle rotates around its tip) and to slip inside tissues along the new direction. Finally, the system updates the equilibrium state proportionally to the amount of the slip. If it equals the final needle depth, the new equilibrium orientation equals the forced orientation, and no update of equilibrium direction is required if no slip occurred.

\subsection{Tissue Penetration}

During tissue penetration, reaction forces are computed by numerical integration, subdividing the needle into a finite number of trunks of constant length and accumulating reaction forces exerted by tissues on the central point of each trunk (see figure 5). The system traverses the volume on the central line of the needle equilibrium position, samples the volume in the central point of each trunk (i.e. extracts from volume a scalar value computed by tri-linear interpolation of the eight nearest voxels) and converts the sample into mechanical parameters via look-up tables. At each trunk's central point, tissue stress is computed as a function of the distance from its equilibrium position and its forced position. Distance is decomposed into a rotational component (deviation from initial direction) and an axial component. Rotational reaction components are used to 
compute elementary torques about the axis through the needle tip and integrated to find a global reaction torque, which equals the torque due to a single force applied to the needle endpoint, i.e. the rotational component of the reaction force sent to the haptic device. Axial distances are used to compute elementary friction forces and are integrated to give a global axial reaction force returned to the haptic device together with the rotational component. When computing friction forces, the system verifies if the static friction threshold is exceeded, in which case it computes the amount of the slip used later in needle equilibrium state update.

When, during needle insertion, the needle tip collides with a new tissue, the position of the separating surface is computed and forces resulting from surface contact, computed as detailed in the previous section, are added to those due to pierced tissues resistance to deformation.

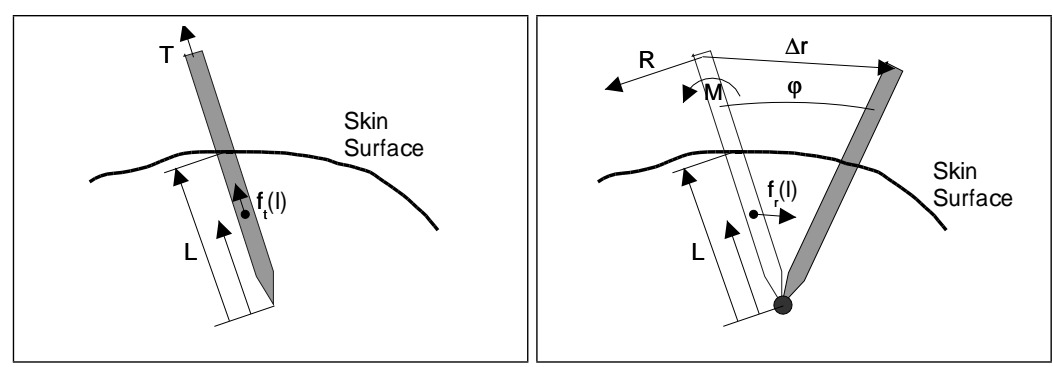

Fig. 5. Translational and rotational reaction components. The total force is equal to $T=$ $\int_{0}^{L} f_{t}(l) d l$ for translation and $M=\tan \phi \int_{0}^{L} l^{2} f_{t}(l) d l$ for rotation. The integrals are numerically evaluated by taking equidistant samples along the needle.

\section{Evaluation}

The system has been repeatedly tested by a vascular surgeon of the S. Elena Hospital, which evaluated the system's behavior when simulating needle insertion into a deep vessel using datasets provided by the Radiology Department of the University Hospital of Pisa. During the test sessions, mechanical properties of the tissues have been tuned interactively, by adjusting parameters one at a time until the response was considered adequate. Test datasets were of size $512 \times 512 \times 128(134 \times 134 \times 190 \mathrm{~mm})$, and were rendered at 10 fps using 256 sampling slices orthogonal to the viewing direction. Force feedback at $1 \mathrm{KHz}$ was generated using 50 samples along the needle. To increase the amount of information provided to users during training sessions, we make it possible to optionally show during interaction a diagram of the forces exerted and an identification of the current tissue traversed by the needle's tip (see figure 6). A five minutes videotape illustrating the system with recordings of live sessions is available [16].

The surgeon's view is that the simulator is sufficiently representative of a real catheter insertion. He appreciates the usefulness of tracked stereoscopic viewing when locating the catheter insertion point, the excellent agreement between visual and tactile 
perception of catheter placement and the realistic haptic response of the system during soft tissues (especially vessel) piercing and penetration. The lack of torque control due to the three degrees-of-freedom force feedback system is not considered a major problem. He judged as less realistic the needle contact with hard tissues like bone, because of the system tendency to generate undesired vibrations when simulating very hard contact with their surface. This situation does not occur in common catheter insertion procedures, but identifies an important problem area in the case of the extension of the system to other procedures (e.g. epidural puncture).

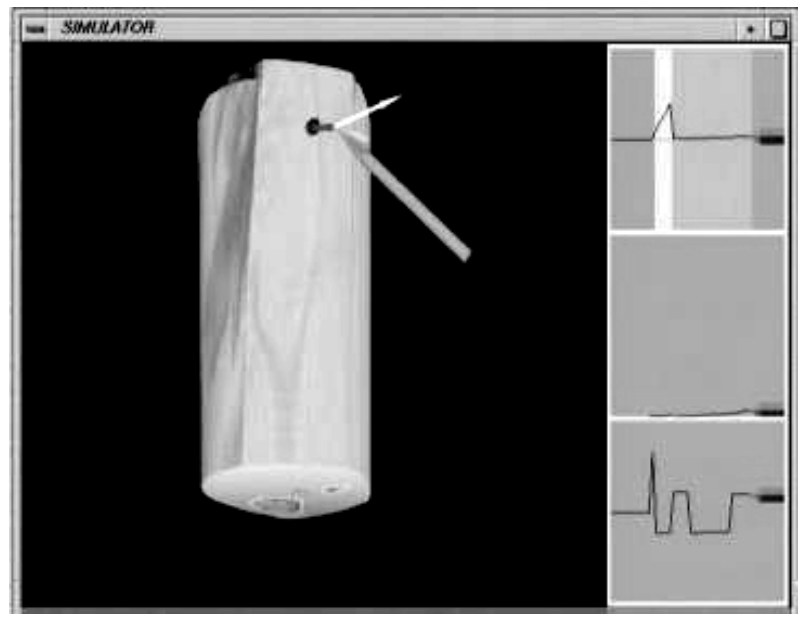

Fig. 6. Display of auxiliary information during the insertion procedure. To increase the amount of information provided, the system optionally shows during interaction a diagram of the forces exerted and an identification of the current tissue traversed by the needle's tip.

\section{Conclusions and Future Work}

We have presented an experimental catheter insertion simulation system that provides users co-registered visual and haptic feedback. The system works on patient-specific volumetric data acquired using standard medical imaging modalities. The actual needle insertion operation is simulated for individual patients, rather than being an example of a model surgical procedure on standard anatomy. Patient specific features may thus be studied in detail by the trainees, overcoming one of the major limitations of current training techniques. Visual and haptic feedback are generated using a direct volume rendering approach, without resorting to surface reconstruction.

The results obtained so far have been encouraging. We are currently in the process to assessing the system's effectiveness through a controlled statistical study with different user groups that compares data acquired running the simulator, e.g. total time needed for insertion, catheter angle at the cutaneous level, traversed tissues and forces over time. 


\section{Acknowledgments}

This work was carried out within research projects IERAPSI (European Union IST Project 12175) and ViVa (partially sponsored by Eureka Project HPPC/SEA - EU 1063). We also acknowledge the contribution of Sardinian regional authorities. Finally, thanks to Andrea Giachetti for support with DICOM images.

\section{References}

1. P. N. Berret, T. J. Parker, A. J. Harrison, T. A. Thomas, and A. Carr. Simulation of resistence forces acting on surgical needles. Proc Instn Mech Engrs, 211(Part H), 1997.

2. Brian Cabral, Nancy Cam, and Jim Foran. Accelerated volume rendering and tomographic reconstruction using texture mapping hardware. In Arie Kaufman and Wolfgang Krueger, editors, 1994 Symposium on Volume Visualization, pages 91-98. ACM SIGGRAPH, October 1994. ISBN 0-89791-741-3.

3. L. Cohen. On active contour models and balloons. CVGIP: Image Understanding, 53(2):211-218, March 1991.

4. Timothy J. Cullip and Ulrich Neumann. Accelerating volume reconstruction with 3D texture hardware. Technical Report TR93-027, Department of Computer Science, University of North Carolina - Chapel Hill, May 11994.

5. Robert A. Drebin, Loren Carpenter, and Pat Hanrahan. Volume rendering. Computer Graphics, 22(4):65-74, August 1988.

6. Enrico Gobbetti, Piero Pili, Antonio Zorcolo, and Massimiliano Tuveri. Interactive virtual angioscopy. In CD-ROM and Video Proceedings IEEE Visualization, Conference held in Research Triangle Park, NC, USA, October 1998 (In press).

7. S. Guan and R. G. Lipes. Innovative volume rendering using 3D texture mapping. In Image Capture, Formatting and Display, volume 2164 of SPIE. SPIE, 1994.

8. M. Kass, A. Witkin, and D. Terzopoulos. Snakes: Active contour models. Int. Journal of Computer Vision, 1:321-331, 1987.

9. Todd Kulick. Building an opengl volume renderer. SGI Dev. News, 1996.

10. Nelson Max. Optical models for direct volume rendering. IEEE Transactions on Visualization and Computer Graphics, 1(2):99-108, June 1995.

11. John S. Montrym, Daniel R. Baum, David L. Dignam, and Christopher J. Migdal. Infinite Reality: a real-time graphics system. Computer Graphics, 31(3A):293-302, August 1997.

12. Luis Serra, Ng Hern, Chua Beng Choon, and Tim Poston. Interactive vessel tracing in volume data. In Proceedings of the 1997 Symposium on Interactive 3D Graphics, April 1997.

13. R. O' Tool, R. Playter, W. Blank, N. Cornelius, W. Roberts, and M. Raibert. A novel virtual reality surgical trainer with force feedback: Surgeon vs. medical student performance. In The Second PHANToM User's Group Proceedings, 1997.

14. Allen Van Gelder and Kwansik Kim. Direct volume rendering with shading via threedimensional textures. In 1996 Volume Visualization Symposium, pages 23-30. IEEE, October 1996. ISBN 0-89791-741-3.

15. Washington University. MIR CTN documentation Version 2.8.3.

16. Antonio Zorcolo, Enrico Gobbetti, and Massimiliano Tuveri. Catheter insertion simulation with combined visual and haptic feedback. Technical Report VIDEOTAPE 99/80, CRS4, Center for Advanced Studies, Research, and Development in Sardinia, Cagliari, Italy, May 1999. Presented at the First PHANToM Users Research Symposium (PURS'99), Heidelberg, Germany, May 21-22. 